Article

\title{
Improvement of Faba Bean Yield Using Rhizobium/Agrobacterium Inoculant in Low-Fertility Sandy Soil
}

\author{
Sameh H. Youseif *, Fayrouz H. Abd El-Megeed and Saleh A. Saleh \\ National Gene Bank and Genetic Resources, Agricultural Research Center, Giza 12619, Egypt; \\ fayrouz_1983@hotmail.com (F.H.A.E.-M.); sasaleh2006@hotmail.com (S.A.S.) \\ * Correspondence: samehheikal@hotmail.com; Tel.: +2-010-1436-2343
}

Academic Editor: Peter Langridge

Received: 19 August 2016; Accepted: 13 December 2016; Published: 1 January 2017

\begin{abstract}
Soil fertility is one of the major limiting factors for crop's productivity in Egypt and the world in general. Biological nitrogen fixation (BNF) has a great importance as a non-polluting and a cost-effective way to improve soil fertility through supplying $\mathrm{N}$ to different agricultural systems. Faba bean (Vicia faba L.) is one of the most efficient nitrogen-fixing legumes that can meet all of their $\mathrm{N}$ needs through BNF. Therefore, understanding the impact of rhizobial inoculation and contrasting soil rhizobia on nodulation and $\mathrm{N}_{2}$ fixation in faba bean is crucial to optimize the crop yield, particularly under low fertility soil conditions. This study investigated the symbiotic effectiveness of 17 Rhizobium/Agrobacterium strains previously isolated from different Egyptian governorates in improving the nodulation and $\mathrm{N}_{2}$ fixation in faba bean cv. Giza 843 under controlled greenhouse conditions. Five strains that had a high nitrogen-fixing capacity under greenhouse conditions were subsequently tested in field trials as faba bean inoculants at Ismaillia Governorate in northeast Egypt in comparison with the chemical N-fertilization treatment $\left(96 \mathrm{~kg} \mathrm{~N} \cdot \mathrm{ha}^{-1}\right)$. A starter N-dose (48 kg N.ha ${ }^{-1}$ ) was applied in combination with different Rhizobium inoculants. The field experiments were established at sites without a background of inoculation under low fertility sandy soil conditions over two successive winter growing seasons, 2012/2013 and 2013/2014. Under greenhouse conditions, inoculated plants produced significantly higher nodules dry weight, plant biomass, and shoot N-uptake than non-inoculated ones. In the first season (2012/2013), inoculation of field-grown faba bean showed significant improvements in seed yield (3.73-4.36 ton $\cdot \mathrm{ha}^{-1}$ ) and seed N-yield (138-153 Kg N.ha $\left.{ }^{-1}\right)$, which were higher than the uninoculated control $\left(48 \mathrm{~kg} \mathrm{~N} \cdot \mathrm{ha}^{-1}\right)$ that produced $2.97 \mathrm{Kg} \cdot \mathrm{ha}^{-1}$ and $95 \mathrm{~kg} \mathrm{~N} \cdot \mathrm{ha}^{-1}$, respectively. Similarly, in the second season (2013/2014), inoculation significantly improved seed yield (3.16-4.68 ton $\cdot \mathrm{ha}^{-1}$ ) and seed $\mathrm{N}$-yield $\left(98-155 \mathrm{Kg} \mathrm{N} \cdot \mathrm{ha}^{-1}\right.$ ) relative to the uninoculated control (48 kg N·ha ${ }^{-1}$ ), which recorded $2.58 \mathrm{Kg} \cdot \mathrm{ha}^{-1}$ and $80 \mathrm{~kg} \mathrm{~N} \cdot \mathrm{ha}^{-1}$, respectively. Interestingly, faba bean inoculated with strain Rlv NGB-FR 126 showed significant increments in seed yield (35\%-48\%) and seed N-yield (34\%-49\%) compared to the inorganic $\mathrm{N}$ fertilizers treatment (96 kg N.ha ${ }^{-1}$ ) over the two cropping seasons, respectively. These results indicate that inoculation of faba bean with effective rhizobial strains can reduce the need for inorganic $\mathrm{N}$ fertilization to achieve higher crop yield under low fertility soil conditions.
\end{abstract}

Keywords: Rhizobium; Agrobacterium; inoculation; soil fertility; faba bean

\section{Introduction}

Faba bean (Vicia faba L., broad bean, horse bean) is a major grain legume widely cultivated in many countries for food and feed purposes [1]. Due to its multiple uses, high nutritional value, and ability to grow over a wide range of climatic and soil conditions, cultivation of faba bean is suitable for sustainable agriculture in many marginal areas [2]. 
Faba bean is one of the oldest legume crops grown in Egypt [3]. However, production has declined considerably from 523,000 tonnes in 1998 to 158,000 tonnes in 2014 [4]—often a result of susceptibility to foliar diseases, the effects of parasites [5], and/or competition with other crops. Egypt now is the world's largest importer of faba bean; its annual requirement of half million tonnes accounts for over half of global imports [6]. Therefore, increasing faba bean production and improving yield quality is a major target to meet the demand of the increasing Egyptian population, since faba bean constitutes a major part of the diet of Egyptian people [7].

Arid land with low nutrient availability, like most of the Egyptian land available for agriculture expansion [8], covers around 30\% of the world's land area [9]. In such poor ecosystems, application of high levels of inorganic fertilizers is a common practice to compensate for nitrogen deficiency which is very costly and is a crucial obstruction toward increasing production of food crops including legumes [10]. In addition, more than $50 \%$ of the applied nitrogen fertilizers are somehow lost through different processes which not only represent a cash loss to the farmers, but also a source of pollution for the environment [11]. Consequently, there has been a growing interest in environmental friendly sustainable agricultural practices [12].

Biological nitrogen fixation, especially rhizobia-legumes symbiosis, is one of the alternative solutions and the promising technologies which play an important role in reducing the consumption of chemical N-fertilizers, increasing soil fertility, decreasing the production cost, and eliminating the undesirable pollution impact of chemical fertilizers in the environment [13]. Worldwide, $\mathrm{N}_{2}$ fixed by nodulated legumes (pulses and oilseeds legumes) is estimated to contribute $21.45 \mathrm{Tg} \mathrm{N}$ annually to global agricultural systems [14].

Like other legumes, faba bean contributes to sustainable agriculture by fixing atmospheric nitrogen in symbiosis with soil rhizobia [15]. Faba bean commonly establishes effective nitrogen fixation symbiosis with fast-growing rhizobia of the species Rhizobium leguminosarum sv. viciae (Rlv) [16]. Later, R. fabae [17], R. laguerrereae [18], R. etli [19,20], and Agrobacterium tumefaciens [20] were also identified as faba bean-nodulating microsymbionts.

Faba bean is one of the most efficient nitrogen-fixing legumes and faba bean plants can meet all of their $\mathrm{N}$ needs through biological nitrogen fixation (BNF) [14,21]. Globally, the amounts of $\mathrm{N}_{2}$-fixed by faba bean were estimated in the range from 45 to $300 \mathrm{~kg} \mathrm{~N} \cdot \mathrm{ha}^{-1}$ [22]. Under different Egyptian field conditions, the amount of $\mathrm{N}_{2}$-fixed by this legume ranged between 121 and $171 \mathrm{~kg} \mathrm{~N} \cdot \mathrm{ha}^{-1}$ [23,24].

Populations of soil rhizobia often vary considerably in their abundance and effectiveness in nodulating and fixing atmospheric nitrogen $\left(\mathrm{N}_{2}\right)$ symbiotically with their legume hosts $[25,26]$. Low fertile soils, particularly sandy soils, contain insufficient numbers of indigenous rhizobia to form efficient symbiotic relationships with their appropriate legumes. In such cases, the reliance on soil rhizobia as the sole source of inoculants can restrict legume yields $[27,28]$. Therefore, research on the impact of legume inoculation with efficient rhizobial strains can assist in defining the potential of inoculation to improve legume yields and increase the contribution of legume fixed $\mathrm{N}$ to the agriculture system [29].

In a previous study, the taxonomic diversity of 42 rhizobial strains that had been isolated from nodules of faba bean grown under different agro-ecological conditions in Egypt was studied using multilocus sequence analyses (MLSA) [20]. Interestingly, only 17 strains were identified as Rlv, while 24 strains were identified as A.tumefaciens, and one strain was classified as R. etli. All isolated strains formed effective symbioses with faba bean plants in Leonard jar assemblies. The present study is complementary to the previous work, and is intended to investigate the potential of highly efficient strains as faba bean inoculants to enhance the crop yield and productivity under low fertility sandy soils compared to the recommended inorganic $\mathrm{N}$-fertilization $\left(96 \mathrm{~kg} \mathrm{~N} \cdot \mathrm{ha}^{-1}\right)$. 


\section{Material and Method}

\subsection{Bacterial Strains}

Seventeen Egyptian strains of faba bean nodulating rhizobia including twelve A. tumefaciens and five R. leguminosarum sv. viciae [20] were used in this study. All strains were grown in yeast extract-mannitol (YEM) medium [30].

\subsection{Symbiotic Effectiveness under Greenhouse Conditions}

Studies of the symbiotic properties (nodulation and nitrogen uptake) of 17 rhizobial strains were conducted with faba bean cultivar Giza 843 in a pot experiment. Plastic pots $(30 \mathrm{~cm}$ diameter) were filled with $10 \mathrm{~kg}$ of sandy soil and arranged in a complete randomized block design with three replicates. Low fertility sandy soil samples with no history of inoculation were collected from Agricultural Research Station, Ismaillia Governorate (30 $37^{\prime} 00.10^{\prime \prime} \mathrm{N}$ and $\left.32^{\circ} 14^{\prime} 38.57^{\prime \prime} \mathrm{E}\right)$. Soil characteristics used in the pot experiment were determined according to [31], and are shown in Table 1. Four seeds were planted in each pot. Each seed was inoculated with $1 \mathrm{~mL}$ of a log phase rhizobial culture $\left(10^{9}\right.$ cells $\left.\mathrm{mL}^{-1}\right)$. Growth conditions of faba bean plants were $12-25{ }^{\circ} \mathrm{C}$ (night/day), a relative humidity of $50 \%-60 \%$, and a photoperiod of $10 \mathrm{hr}$. At flowering stage, 50 days after sowing, plants were uprooted and assayed for dry weight of nodules, shoots and roots dry weight, as well as shoot N-uptake by faba bean plants.

Table 1. Physical and chemical properties of different sandy soils used in this study.

\begin{tabular}{|c|c|c|c|}
\hline \multirow[b]{2}{*}{ Property } & \multicolumn{3}{|c|}{ Value } \\
\hline & $\begin{array}{l}\text { Greenhouse } \\
\text { Experiment }\end{array}$ & $\begin{array}{l}\text { Field Winter Growing } \\
\text { Season } 2012 / 2013\end{array}$ & $\begin{array}{l}\text { Field Winter Growing } \\
\text { Season } 2013 / 2014\end{array}$ \\
\hline Texture grade & Sandy & Sandy & Sandy \\
\hline $\mathrm{CaCo}_{3}(\%)$ & 2.85 & 2.80 & 1.95 \\
\hline Saturation percent S.P (\%) & 19.60 & 27.90 & 27.60 \\
\hline $\mathrm{pH}$ & 7.75 & 8.20 & 7.94 \\
\hline $\begin{array}{l}\text { Electrical conductivity } \\
\left(\mathrm{dS} \cdot \mathrm{m}^{-1} \text { at } 25^{\circ} \mathrm{C}\right)\end{array}$ & 0.67 & 0.90 & 0.81 \\
\hline \multicolumn{4}{|l|}{ Soluble cations (meq/L) } \\
\hline $\mathrm{Ca}^{+2}$ & 2.12 & 3.40 & 3.00 \\
\hline $\mathrm{Mg}^{+2}$ & 1.10 & 1.30 & 1.18 \\
\hline $\mathrm{Na}^{+}$ & 2.57 & 5.30 & 3.61 \\
\hline $\mathrm{K}^{+}$ & 1.10 & 0.40 & 0.35 \\
\hline \multicolumn{4}{|l|}{ Soluble anions (meq/L) } \\
\hline $\mathrm{CO}_{3}^{-2}$ & 0.00 & 0.00 & 0.00 \\
\hline $\mathrm{HCO}_{3}{ }^{-}$ & 1.95 & 1.20 & 1.10 \\
\hline $\mathrm{Cl}^{-}$ & 3.00 & 3.40 & 3.10 \\
\hline $\mathrm{SO}_{4}^{-2}$ & 1.94 & 5.80 & 3.94 \\
\hline Total N $(\%)$ & 0.017 & 0.024 & 0.021 \\
\hline Total Soluble-N $\left(\mathrm{mg} \cdot \mathrm{Kg}^{-1}\right)$ & 8.50 & 20.00 & 19.50 \\
\hline Available-P $\left(\mathrm{mg} \cdot \mathrm{Kg}^{-1}\right)$ & 3.10 & 4.90 & 4.45 \\
\hline Available-K $\left(\mathrm{mg} \cdot \mathrm{Kg}^{-1}\right)$ & 179.00 & 252.00 & 238.50 \\
\hline Organic matter $(\%)$ & 0.31 & 0.31 & 0.30 \\
\hline \multicolumn{4}{|l|}{$\begin{array}{l}\text { Available micronutrients } \\
\qquad\left(\mathrm{mg} \cdot \mathrm{Kg}^{-1}\right)\end{array}$} \\
\hline $\mathrm{Fe}$ & 1.30 & 4.30 & 3.92 \\
\hline $\mathrm{Mn}$ & 0.90 & 2.10 & 1.95 \\
\hline $\mathrm{Zn}$ & 1.00 & 1.20 & 1.35 \\
\hline $\mathrm{Cu}$ & 0.03 & 0.02 & 0.05 \\
\hline
\end{tabular}




\subsection{Field Experiments}

Field experiments were carried out at El Wasfeya village, Ismaillia Governorate, Egypt $\left(30^{\circ} 34^{\prime} 27.30^{\prime \prime} \mathrm{N}, 32^{\circ} 10^{\prime} 26.21^{\prime \prime} \mathrm{E}\right)$ in the two successive winter growing seasons, $2012 / 2013$ and 2013/2014. Soil characteristics from experimental sites were determined according to [31]. The main physical and chemical properties of the soil are shown in Table 1. Faba bean variety Giza 843 was used due to its tolerance to drought stress conditions. Faba bean seeds were sown in the rate of $100 \mathrm{~kg}$ seeds ha ${ }^{-1}$ and were cultivated in strips. Each strip $(4 \mathrm{~m} \times 12 \mathrm{~m})$ consisted of four plots. Each plot area was $12 \mathrm{~m}^{2}$ and consisted of four rows, spaced $0.6 \mathrm{~m}$ apart. An additional fifth row was placed in each plot and served as a border, and was not involved in calculations. Each strip was spaced apart by $1 \mathrm{~m}$ apart to prevent bacterial migrations. Weeds, insects, and fungal pathogens were controlled by chemical spray applications, as required, at rates according to manufacturers' recommendations. At flowering stage, 50 days after sowing, plant samples from each plot were randomly selected from as uniform of an area as possible (in the middle of the second and third lines), in order to avoid heterogeneous conditions or disturbed sites, for estimating nodules dry weight, plant dry matters, and shoot $\mathrm{N}$-content. At harvest, biological yield was determined by the mechanical harvesting of the entire plot using a plot harvester.

\subsection{Fertilization}

All treatments received the recommended dose of phosphate and potassium fertilization in the rate of $75 \mathrm{~kg} \mathrm{P}_{2} \mathrm{O}_{5} \mathrm{ha}^{-1}$ and $115 \mathrm{~kg} \mathrm{~K}_{2} \mathrm{O} \mathrm{ha}^{-1}$, respectively. All bacterial treatments received $48 \mathrm{~kg} \mathrm{~N} \cdot \mathrm{ha}^{-1}$ as a starter $\mathrm{N}$-dose. In addition, three un-inoculated controls were involved; $\left(\mathrm{T}_{0}\right)$ the uninoculated non-N fertilized control; $(\mathrm{T})$ the uninoculated with starter N-dose $\left(48 \mathrm{~kg} \mathrm{~N} \cdot \mathrm{ha}^{-1}\right)$; and $(\mathrm{TN})$ the uninoculated with full $\mathrm{N}$-fertilizers $\left(96 \mathrm{~kg} \mathrm{~N} \cdot \mathrm{ha}^{-1}\right)$.

\subsection{Inocula Preparation and Seed Inoculation}

Vermiculite supplemented with $10 \%$ peat was used as a powder carrier [32], packed in polyethylene bags ( $300 \mathrm{~g}$ carrier per bag), sealed and sterilized by gamma irradiation $\left(2.5 \times 10^{6}\right.$ rads). Rhizobial strains were grown in YEM medium [30]. Cultures of $\left(1 \times 10^{9}\right.$ colony-forming unit $\left.\mathrm{mL}^{-1}\right)$ were injected into the carrier to satisfy $60 \%$ of water holding capacity. At sowing, faba bean seeds were coated with different rhizobial inoculants at a rate of $10 \mathrm{~g}$ of inoculant $/ 1 \mathrm{~kg}$ seeds, using Arabic gum solution (16\%) as the adhesive agent for seed coating [33].

\subsection{Statistical Analysis}

Data was analyzed for variance using the MSTAT analysis software [34].

\section{Results}

\subsection{Symbiotic Effectiveness under Greenhouse Conditions}

The symbiotic efficiency of 17 rhizobial strains related to Rlv and A. tumefaciens was assessed with faba bean cv. Giza 843 in a pot experiment under greenhouse conditions. The effect of inoculation on dry weight of nodules, plant dry weight accumulation, and shoot N-content is shown in Table 2 . All strains successfully nodulated faba bean and showed different nodulation patterns which ranged from 82 to $366 \mathrm{mg}$ nodules/plant (Table 2). Rlv strains NGB-FR 126 and NGB-FR 128 resulted in the highest dry weight of nodules with 366 and $295 \mathrm{mg}$ nodules/plant, respectively. In case of A. tumefaciens strains, the maximum dry weight of nodules ( $230 \mathrm{mg}$ nodules/plant) was produced by strain NGB-FR 39. Nevertheless, the uninoculated controls $\mathrm{T}_{0}, \mathrm{~T}$, and TN resulted in 48, 63 and $52 \mathrm{mg}$ nodules/plant, respectively. Shoot dry weight of faba bean plants increased significantly in response to effective inoculation, however, no significant variations were observed for root dry weight. Out of the tested strains, eight strains (NGB-FR 39, 62, 65, 70, 107, 126, 128, and 142), resulted in significant 
increment in shoot dry matter, which ranged from 4.01 to $4.27 \mathrm{~g} /$ plant relative to the uninoculated control (T) with $48 \mathrm{~kg} \mathrm{~N} \cdot \mathrm{ha}^{-1}$. In the same trend, shoot N-content was clearly affected according to the type of inoculated strains (Table 2). All inoculated strains, except for NGB-FR 26 and 51, produced shoot $\mathrm{N}$ content significantly higher than that obtained with non-inoculated control (T). Faba bean plants inoculated by Rlv strain NGB-FR 126 showed the highest shoot N-content (152 mg N/plant) which was significant greater than all other treatments, including the full $\mathrm{N}$-fertilized treatment (TN), which recorded $142 \mathrm{mg} \mathrm{N} /$ plant (Table 2). On the other hand, all inoculated strains except for strain NGB-FR 26 significantly increased shoot dry weight and accumulated higher $\mathrm{N}$ in plants than the uninoculated non-fertilized control $\left(\mathrm{T}_{0}\right)$.

Table 2. List of faba bean nodulating rhizobia used in this study, their identity ${ }^{*}$, and symbiotic properties under greenhouse condition.

\begin{tabular}{|c|c|c|c|c|c|c|c|c|c|}
\hline \multirow{3}{*}{$\begin{array}{l}\text { Treatment } \\
\text { NGB-FR } 10\end{array}$} & \multirow{2}{*}{ Identity * } & \multirow{2}{*}{\multicolumn{2}{|c|}{$\begin{array}{l}\text { Dry Weight of } \\
\text { Nodules (mg) }\end{array}$}} & \multicolumn{4}{|c|}{ Dry wt. (g/Plant) } & \multirow{2}{*}{\multicolumn{2}{|c|}{$\begin{array}{l}\text { Shoot N Content } \\
\text { (mg N/Plant) }\end{array}$}} \\
\hline & & & & \multicolumn{2}{|c|}{ Root } & \multicolumn{2}{|c|}{ Shoot } & & \\
\hline & A. tumefaciens & 103 & $\mathrm{~g}$ & 1.68 & $\mathrm{a}$ & 3.62 & cdefg & 122 & hi \\
\hline NGB-FR 25 & A. tumefaciens & 82 & hi & 1.57 & $\mathrm{a}$ & 3.61 & defg & 123 & $\mathrm{~h}$ \\
\hline NGB-FR 26 & A. tumefaciens & 82 & hi & 1.49 & a & 3.40 & fgh & 109 & 1 \\
\hline NGB-FR 27 & A. tumefaciens & 96 & $\mathrm{gh}$ & 1.54 & $\mathrm{a}$ & 3.83 & abcdefg & 130 & $\mathrm{~g}$ \\
\hline NGB-FR 39 & A. tumefaciens & 230 & $\mathrm{~d}$ & 1.80 & $\mathrm{a}$ & 4.18 & abcde & 142 & de \\
\hline NGB-FR 51 & A. tumefaciens & 94 & $\mathrm{gh}$ & 1.47 & a & 3.62 & cdefg & 114 & $\mathrm{k}$ \\
\hline NGB-FR 62 & A. tumefaciens & 130 & $\mathrm{f}$ & 1.68 & a & 4.27 & $a b^{\circ}$ & 145 & $\mathrm{bc}$ \\
\hline NGB-FR 65 & R. leguminosarum sv. viciae & 172 & e & 1.78 & a & 4.16 & abcde & 141 & de \\
\hline NGB-FR 70 & R. leguminosarum sv. viciae & 191 & e & 1.79 & a & 4.22 & abcd & 139 & ef \\
\hline NGB-FR 99 & A. tumefaciens & 104 & $\mathrm{~g}$ & 1.49 & a & 3.67 & bcdefg & 118 & j \\
\hline NGB-FR 107 & A. tumefaciens & 100 & $\mathrm{gh}$ & 1.78 & $\mathrm{a}$ & 4.01 & abcdef & 137 & $\mathrm{f}$ \\
\hline NGB-FR 122 & A. tumefaciens & 182 & $\mathrm{e}$ & 1.62 & $\mathrm{a}$ & 3.82 & abcdefg & 130 & $\mathrm{~g}$ \\
\hline NGB-FR 126 & R. leguminosarum sv. viciae & 366 & a & 1.94 & $\mathrm{a}$ & 4.26 & $\mathrm{ab}$ & 152 & $\mathrm{a}$ \\
\hline NGB-FR 128 & R. leguminosarum sv. viciae & 295 & $\mathrm{~b}$ & 1.89 & $\mathrm{a}$ & 4.24 & $a b c$ & 144 & $\mathrm{~cd}$ \\
\hline NGB-FR 132 & A. tumefaciens & 98 & $\mathrm{gh}$ & 1.60 & $\mathrm{a}$ & 3.60 & efg & 119 & ij \\
\hline NGB-FR 140 & R. leguminosarum sv. viciae & 146 & $\mathrm{f}$ & 1.87 & $\mathrm{a}$ & 3.82 & abcdefg & 122 & hi \\
\hline NGB-FR 142 & A. tumefaciens & 130 & $\mathrm{f}$ & 1.60 & $\mathrm{a}$ & 4.13 & abcde & 136 & $\mathrm{f}$ \\
\hline $\mathrm{T}_{0}$ & & 48 & j & 1.32 & $\mathrm{a}$ & 2.87 & $\mathrm{~h}$ & 89 & $\mathrm{~m}$ \\
\hline $\mathrm{T}$ & & 63 & ij & 1.46 & a & 3.34 & $\mathrm{gh}$ & 114 & k \\
\hline $\mathrm{TN}$ & & 52 & $j$ & 1.91 & a & 4.32 & $\mathrm{a}$ & 142 & cde \\
\hline
\end{tabular}

* Bacterial identification based on 16S rRNA sequencing and multilocus sequence typing [20]. Data per plant are means of four replicates (three plants per replicate). Values followed by the same letter within each column are not significantly different at $p<0.05$. $\mathrm{T}_{0}$ : uninoculated seeds and non-chemical $\mathrm{N}$-fertilizers. T: uninoculated seeds plus starter N-fertilizer $\left(48 \mathrm{~kg} \mathrm{~N} \cdot \mathrm{ha}^{-1}\right)$. TN: uninoculated seeds and full N-fertilizer $\left(96 \mathrm{~kg} \mathrm{~N} \cdot \mathrm{ha}^{-1}\right)$.

\subsection{Evaluation of Faba Bean Inoculation under Field Trials}

Two field experiments were conducted over two successive growing seasons (2012/2013 and 2013/2014), in low fertility sandy soils at Ismaillia Governorate in order to investigate the symbiotic properties of the rhizobial strains that had a high nitrogen-fixing capacity under controlled conditions in the greenhouse. The results showed that all selected strains were able to nodulate faba bean cv. Giza 843 under field-grown conditions (Tables 3-6).

In the first season (2012/2013), at flowering stage, the highest nodules dry mass per plant was achieved by Rlv NGB-FR 128 (367 mg/plant) followed by Rlv NGB-FR 126 (322 mg/plant), which was significantly higher than that obtained by other tested strains (Table 3). On the other hand, the uninoculated controls $\left(\mathrm{T}_{0}, \mathrm{~T}\right.$, and $\left.\mathrm{TN}\right)$ showed nodulation status ranged from 11-83 mg nodules/plant. Highly significant differences were observed in the dry matter of faba bean plants according to different rhizobial inoculations (Table 3). Faba bean plants inoculated by Rlv NGB-FR 126 and A. tumefaciens NGB-FR 62 showed the highest shoot dry weights (6.2 and $6.1 \mathrm{~g} /$ plant, respectively), which were significantly higher relative to the full N-uninoculated control (TN) that resulted in $5.4 \mathrm{~g} /$ plant. Similarly, Rlv NGB-FR 126 resulted in the maximum shoot N-content (281 mg N/plant), which was 
significantly greater as compared to the uninoculated controls where the accumulated $\mathrm{N}$ in shoots ranged from $128-258 \mathrm{mg} \mathrm{N} /$ plant.

Table 3. Effect of different rhizobial strains on nodulation, growth parameters, and shoot $\mathrm{N}$ content of faba bean plants after 50 days of sowing under field conditions (winter growing season 2012/2013).

\begin{tabular}{lcccccccc}
\hline \multirow{2}{*}{ Treatment } & \multirow{2}{*}{$\begin{array}{c}\text { Dry wt. of Nodules } \\
\text { (mg/Plant) }\end{array}$} & \multicolumn{3}{c}{ Dry wt. (g/Plant) } & \multicolumn{2}{c}{$\begin{array}{c}\text { Shoot N Content } \\
\text { (mg N/Plant) }\end{array}$} \\
\cline { 5 - 7 } & & \multicolumn{2}{c}{ Root } & \multicolumn{2}{c}{ Shoot } & & \\
\hline NGB-FR-39 & 85 & $\mathrm{e}$ & 0.84 & $\mathrm{~d}$ & 4.3 & $\mathrm{e}$ & 172 & $\mathrm{~d}$ \\
NGB-FR-62 & 287 & $\mathrm{c}$ & 1.21 & $\mathrm{a}$ & 6.1 & $\mathrm{ab}$ & 256 & $\mathrm{~b}$ \\
NGB-FR-70 & 143 & $\mathrm{~d}$ & 1.08 & $\mathrm{~b}$ & 4.7 & $\mathrm{e}$ & 181 & $\mathrm{~d}$ \\
NGB-FR-126 & 322 & $\mathrm{~b}$ & 1.20 & $\mathrm{a}$ & 6.2 & $\mathrm{a}$ & 281 & $\mathrm{a}$ \\
NGB-FR-128 & 367 & $\mathrm{a}$ & 1.14 & $\mathrm{ab}$ & 5.5 & $\mathrm{bc}$ & 251 & $\mathrm{~b}$ \\
$\mathrm{~T}_{0}$ & 28 & $\mathrm{f}$ & 0.69 & $\mathrm{e}$ & 3.1 & $\mathrm{f}$ & 128 & $\mathrm{e}$ \\
$\mathrm{T}$ & 83 & $\mathrm{e}$ & 0.96 & $\mathrm{c}$ & 4.8 & $\mathrm{de}$ & 211 & $\mathrm{c}$ \\
$\mathrm{TN}$ & 11 & $\mathrm{f}$ & 1.19 & $\mathrm{a}$ & 5.4 & $\mathrm{~cd}$ & 258 & $\mathrm{~b}$ \\
\hline
\end{tabular}

Data per plant are means of four replicates (three plants per replicate). Values followed by the same letter within each column are not significantly different at $p<0.05 . \mathrm{T}_{0}$ : uninoculated seeds and non-chemical $\mathrm{N}$-fertilizers. T: uninoculated seeds plus starter $\mathrm{N}$-fertilizer $\left(48 \mathrm{~kg} \mathrm{~N} \cdot \mathrm{ha}^{-1}\right)$. TN: uninoculated seeds and full $\mathrm{N}$-fertilizer $\left(96 \mathrm{~kg} \mathrm{~N} \cdot \mathrm{ha}^{-1}\right)$.

Table 4. Effect of different rhizobial strains on different growth parameters and yield of faba bean plants under field conditions (winter growing season 2012/2013).

\begin{tabular}{|c|c|c|c|c|c|c|c|c|c|c|c|c|c|c|c|c|}
\hline \multirow{3}{*}{$\begin{array}{l}\text { Treatment } \\
\text { NGB-FR-39 }\end{array}$} & \multirow{2}{*}{\multicolumn{2}{|c|}{$\begin{array}{c}\text { Plant } \\
\text { Height } \\
\text { (cm) }\end{array}$}} & \multirow{2}{*}{\multicolumn{2}{|c|}{$\begin{array}{c}\text { No of } \\
\text { Branches/ } \\
\text { Plant }\end{array}$}} & \multirow{2}{*}{\multicolumn{2}{|c|}{$\begin{array}{c}\text { No of } \\
\text { Pods/Plant }\end{array}$}} & \multirow{2}{*}{\multicolumn{2}{|c|}{$\begin{array}{c}\text { No of } \\
\text { Seeds/Plant }\end{array}$}} & \multirow{2}{*}{\multicolumn{2}{|c|}{$\begin{array}{l}\text { Seed } \\
\text { Index }\end{array}$}} & \multicolumn{4}{|c|}{ Yield (ton/ha) } & \multirow{2}{*}{\multicolumn{2}{|c|}{$\begin{array}{c}\text { Seed N } \\
\text { Yield } \\
\text { kgN/ha }\end{array}$}} \\
\hline & & & & & & & & & & & \multicolumn{2}{|c|}{ Straw } & \multicolumn{2}{|c|}{ Seed } & & \\
\hline & 89 & $\mathrm{~d}$ & 3.0 & $\mathrm{a}$ & 18 & $\mathrm{~cd}$ & 48 & de & 75.7 & c & 3.6 & bcd & 2.83 & $\mathrm{~d}$ & 85 & $\mathrm{e}$ \\
\hline NGB-FR-62 & 127 & $\mathrm{~b}$ & 3.3 & $\mathrm{a}$ & 22 & $\mathrm{ab}$ & 65 & $\mathrm{ab}$ & 79.2 & $\mathrm{a}$ & 4.6 & $\mathrm{ab}$ & 4.29 & a & 150 & $\mathrm{a}$ \\
\hline NGB-FR-70 & 90 & $\mathrm{~d}$ & 3.3 & $\mathrm{a}$ & 15 & de & 43 & $\mathrm{e}$ & 72.3 & $\mathrm{~d}$ & 2.9 & $\mathrm{~cd}$ & 2.78 & d & 83 & $\mathrm{e}$ \\
\hline NGB-FR-126 & 134 & a & 3.7 & $\mathrm{a}$ & 24 & $\mathrm{a}$ & 72 & $\mathrm{a}$ & 79.0 & $\mathrm{a}$ & 5.9 & $\mathrm{a}$ & 4.36 & a & 153 & a \\
\hline NGB-FR-128 & 128 & $b$ & 3.3 & $\mathrm{a}$ & 20 & bc & 61 & bc & 78.3 & $\mathrm{ab}$ & 4.4 & $\mathrm{~b}$ & 3.73 & $b$ & 138 & $b$ \\
\hline $\mathrm{T}_{0}$ & 72 & $\mathrm{e}$ & 3.0 & $\mathrm{a}$ & 13 & $\mathrm{e}$ & 31 & $\mathrm{f}$ & 69.2 & $\mathrm{e}$ & 2.4 & $\mathrm{~d}$ & 1.76 & $\mathrm{e}$ & 53 & $\mathrm{f}$ \\
\hline $\mathrm{T}$ & 92 & $\mathrm{~d}$ & 3.3 & $\mathrm{a}$ & 19 & bc & 53 & $\mathrm{~cd}$ & 76.1 & bc & 3.8 & bc & 2.97 & $\mathrm{~d}$ & 95 & $\mathrm{~d}$ \\
\hline $\mathrm{TN}$ & 101 & c & 3.7 & $\mathrm{a}$ & 22 & $\mathrm{ab}$ & 62 & bc & 78.1 & $a b c$ & 4.5 & $\mathrm{ab}$ & 3.24 & c & 114 & C \\
\hline
\end{tabular}

Values followed by the same letter within each column are not significantly different at $p<0.05$. $\mathrm{T}_{0}$ : uninoculated seeds and non-chemical N-fertilizers. T: uninoculated seeds plus starter N-fertilizer $\left(48 \mathrm{~kg} \mathrm{~N} \cdot \mathrm{ha}^{-1}\right)$. TN: uninoculated seeds and full N-fertilizer $\left(96 \mathrm{~kg} \mathrm{~N} \cdot \mathrm{ha}^{-1}\right)$.

Table 5. Effect of different rhizobial strains on nodulation, growth parameters, and shoot $\mathrm{N}$ content of faba bean plants after 50 days of sowing under field conditions (winter growing season 2013/2014).

\begin{tabular}{lcccccccc}
\hline \multirow{2}{*}{ Treatment } & \multirow{2}{*}{$\begin{array}{c}\text { Dry wt. of Nodules } \\
\text { (mg/Plant) }\end{array}$} & \multicolumn{3}{c}{ Dry wt. (g/plant) } & \multicolumn{2}{c}{$\begin{array}{c}\text { Shoot N Content } \\
\text { (mgN/Plant) }\end{array}$} \\
\cline { 4 - 7 } & & \multicolumn{2}{c}{ Root } & \multicolumn{2}{c}{ Shoot } & & & \\
\hline NGB-FR-39 & 309 & $\mathrm{e}$ & 1.86 & $\mathrm{c}$ & 10.4 & $\mathrm{c}$ & 312 & $\mathrm{c}$ \\
NGB-FR-62 & 421 & $\mathrm{~d}$ & 1.86 & $\mathrm{c}$ & 10.2 & $\mathrm{c}$ & 307 & $\mathrm{c}$ \\
NGB-FR-70 & 711 & $\mathrm{~b}$ & 2.32 & $\mathrm{~b}$ & 12.9 & $\mathrm{~b}$ & 402 & $\mathrm{~b}$ \\
NGB-FR-126 & 814 & $\mathrm{a}$ & 2.53 & $\mathrm{a}$ & 14.9 & $\mathrm{a}$ & 483 & $\mathrm{a}$ \\
NGB-FR-128 & 581 & $\mathrm{c}$ & 1.73 & $\mathrm{c}$ & 9.4 & $\mathrm{~d}$ & 283 & $\mathrm{~d}$ \\
$\mathrm{~T}_{0}$ & 19 & $\mathrm{f}$ & 1.10 & $\mathrm{e}$ & 5.8 & $\mathrm{f}$ & 151 & $\mathrm{f}$ \\
$\mathrm{T}$ & 59 & $\mathrm{f}$ & 1.51 & $\mathrm{~d}$ & 8.3 & $\mathrm{e}$ & 233 & $\mathrm{e}$ \\
TN & 14 & $\mathrm{f}$ & 1.80 & $\mathrm{c}$ & 9.5 & $\mathrm{~d}$ & 285 & $\mathrm{~d}$ \\
\hline
\end{tabular}

Data per plant are means of four replicates (three plants per replicate). Values followed by the same letter within each column are not significantly different at $p<0.05$. $\mathrm{T}_{0}$ : uninoculated seeds and non-chemical N-fertilizers.

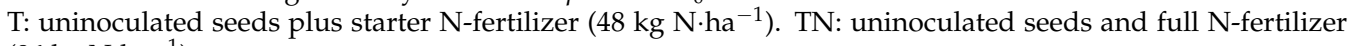
$\left(96 \mathrm{~kg} \mathrm{~N} \cdot \mathrm{ha}^{-1}\right)$. 
Table 6. Effect of different rhizobial strains on different growth parameters and yield of faba bean plants under field conditions (winter growing season 2013/2014).

\begin{tabular}{|c|c|c|c|c|c|c|c|c|c|c|c|c|c|c|c|c|}
\hline \multirow{3}{*}{$\begin{array}{l}\text { Treatment } \\
\text { NGB-FR-39 }\end{array}$} & \multirow{2}{*}{\multicolumn{2}{|c|}{$\begin{array}{l}\text { Plant } \\
\text { Height } \\
\text { (cm) }\end{array}$}} & \multirow{2}{*}{\multicolumn{2}{|c|}{$\begin{array}{c}\text { No of } \\
\text { Branches/ } \\
\text { Plant }\end{array}$}} & \multirow{2}{*}{\multicolumn{2}{|c|}{$\begin{array}{c}\text { No of } \\
\text { Pods/Plant }\end{array}$}} & \multirow{2}{*}{\multicolumn{2}{|c|}{$\begin{array}{c}\text { No of } \\
\text { Seeds/Plant }\end{array}$}} & \multirow{2}{*}{\multicolumn{2}{|c|}{$\begin{array}{l}\text { Seed } \\
\text { Index }\end{array}$}} & \multicolumn{4}{|c|}{ Yield (ton/ha) } & \multirow{2}{*}{\multicolumn{2}{|c|}{$\begin{array}{c}\text { Seed N } \\
\text { Yield } \\
\mathrm{kgN} / \mathrm{ha}\end{array}$}} \\
\hline & & & & & & & & & & & \multicolumn{2}{|c|}{ Straw } & \multicolumn{2}{|c|}{ Seed } & & \\
\hline & 125 & c & 4.5 & $\mathrm{a}$ & 18 & $\mathrm{~b}$ & 52 & $\mathrm{~b}$ & 90.5 & bcd & 6.6 & c & 3.51 & $\mathrm{~b}$ & 109 & b \\
\hline NGB-FR-62 & 123 & $\mathrm{~cd}$ & 4.3 & $a b$ & 17 & $\mathrm{bc}$ & 45 & bc & 92.3 & $a b c$ & 6.5 & c & 3.43 & bc & 107 & b \\
\hline NGB-FR-70 & 150 & $\mathrm{~b}$ & 4.5 & $\mathrm{a}$ & 20 & $\mathrm{a}$ & 61 & $\mathrm{a}$ & 93.0 & $\mathrm{ab}$ & 8.4 & $\mathrm{~b}$ & 4.35 & $\mathrm{a}$ & 148 & a \\
\hline NGB-FR-126 & 173 & $\mathrm{a}$ & 4.5 & a & 21 & a & 64 & $\mathrm{a}$ & 95.3 & $\mathrm{a}$ & 8.9 & a & 4.68 & $\mathrm{a}$ & 155 & a \\
\hline NGB-FR-128 & 116 & d & 4.0 & $a b$ & 14 & d & 40 & $\mathrm{~cd}$ & 89.0 & $\mathrm{~cd}$ & 6.1 & d & 3.16 & c & 98 & b \\
\hline $\mathrm{T}_{0}$ & 80 & $\mathrm{f}$ & 3.0 & c & 8 & e & 25 & $\mathrm{e}$ & 81.3 & $\mathrm{f}$ & 3.4 & $\mathrm{f}$ & 1.58 & $\mathrm{e}$ & 47 & d \\
\hline $\mathrm{T}$ & 102 & $\mathrm{e}$ & 3.5 & bc & 13 & $\mathrm{~d}$ & 32 & de & 84.8 & e & 5.3 & e & 2.58 & d & 80 & c \\
\hline $\mathrm{TN}$ & 117 & $\mathrm{~cd}$ & 4.0 & $a b$ & 15 & $\mathrm{~cd}$ & 38 & $\mathrm{~cd}$ & 88.5 & d & 6.0 & d & 3.17 & c & 104 & b \\
\hline
\end{tabular}

Values followed by the same letter within each column are not significantly different at $p<0.05$. $\mathrm{T}_{0}$ : uninoculated seeds and non-chemical $\mathrm{N}$-fertilizers. T: uninoculated seeds plus starter $\mathrm{N}$-fertilizer $\left(48 \mathrm{~kg} \mathrm{~N} \cdot \mathrm{ha}^{-1}\right)$. TN: uninoculated seeds and full N-fertilizer $\left(96 \mathrm{~kg} \mathrm{~N} \cdot \mathrm{ha}^{-1}\right)$.

At harvest, in the first season (2012/2013), rhizobial inoculation induced significant increases in plant height, number of pods/plant, number of seeds/plant, and seed index of faba beans according to the type of inoculated strain (Table 4). Inoculated plants with strains NGB-FR 62, 126, and 128 enhanced plant height (127-134 cm), which was significantly higher than the uninoculated control plants (T). Likewise, inoculation with strain NGB-FR 126 produced the highest number of pods/plant (24 pods), which was significantly greater than the uninoculated control $(\mathrm{T})$, that produced 19 pods/plant. Similarly, strains NGB-FR 62 and 126 gave the maximum number of seeds/plant and seed index, which were significantly higher than the uninoculated control (T). On the other hand, number of branches had no significant variations among tested strains and the uninoculated controls $\left(\mathrm{T}_{0}, \mathrm{~T}\right.$, and $\left.\mathrm{TN}\right)$. Faba bean inoculated with Rlv NGB-FR 126 and A. tumefaciens NGB-FR 62 produced the maximum seed yield (4.36 and $4.29 \mathrm{ton} \cdot \mathrm{ha}^{-1}$, respectively) and the maximum seed N-yield (153 and $150 \mathrm{~kg} \mathrm{~N} \cdot \mathrm{ha}^{-1}$, respectively), which were significantly higher compared to the full-N fertilized uninoculated control (TN).

In the second season 2013/2014, at flowering stage, the significant effect of rhizobial inoculations on nodulation and plant growth parameters was obvious (Table 5). Strain Rlv NGB-FR 126 produced the highest dry weight of nodules ( $814 \mathrm{mg}$ nodules/plant), while the uninoculated controls $\left(\mathrm{T}_{0}, \mathrm{~T}\right.$, and TN) gave nodulations with a range of $14-59 \mathrm{mg}$ nodules/plant. Faba bean plants inoculated with Rlv NGB-FR 126 recorded the maximum root dry weight $(2.53 \mathrm{~g} /$ plant), shoot dry weight (14.9 g/plant), and shoot $\mathrm{N}$-content (483 mg N/plant) with significant increases higher than the full $\mathrm{N}$-fertilizer uninoculated control (TN), which resulted in $1.8 \mathrm{~g} /$ plant, $9.5 \mathrm{~g} /$ plant, and $285 \mathrm{mg} \mathrm{N} /$ plant, respectively (Table 5).

At harvest, plant height was significantly increased upon inoculation by all tested strains, which was greater than the uninoculated control $(\mathrm{T})$. In the same trend, inoculation by all rhizobial strains, except in case of NGB-FR 128, produced significant increments in number of pods/plant (17-21 pods) and number of seeds/plant (45-64 seeds) relative to the uninoculated control (T) which gave 13 pods/plant and 32 seeds/plant, respectively. Crop yield and seed N-yield of the inoculated faba bean in the second season $(2013 / 2014)$ surpassed those obtained by the un-inoculated controls (Table 6). All over again, the capacity of Rlv NGB-FR 126 to produce the uppermost seed yield (4.68 ton $\cdot \mathrm{ha}^{-1}$ ) and seed $\mathrm{N}$-yield $\left(155 \mathrm{~kg} \mathrm{~N} \cdot \mathrm{ha}^{-1}\right.$ ) was confirmed and was significantly greater than the full-N fertilizers treatment (TN), which recorded $3.17 \mathrm{ton} \cdot \mathrm{ha}^{-1}$ and $104 \mathrm{~kg} \mathrm{~N} \cdot \mathrm{ha}^{-1}$, respectively (Table 6).

Over the two experimental seasons, seed inoculation and nitrogen fertilization treatments (48 and $96 \mathrm{~kg} \mathrm{~N} \cdot \mathrm{ha}^{-1}$ ) produced significantly higher seed yield and seed N-yield compared to non-inoculated non-fertilized control $\left(\mathrm{T}_{0}\right)$, indicating that $\mathrm{N}$ availability under such low fertility soil is a major constraint for crop productivity. 


\section{Discussion}

Low fertility of soil is one of the major constraints limiting crop productivity [8]. The success of legume grain crops is dependent on their capacity to form effective nitrogen-fixing symbioses with root-nodule bacteria. However, many soils may do not have adequate amounts of native rhizobia in terms of number, quality, or effectiveness to enhance biological nitrogen fixation [29]. Rhizobium-legume association can be manipulated, through inoculation under N-limiting field conditions, to improve crop production easily and inexpensively [35]. Where natural $\mathrm{N}_{2}$ fixation is not optimal, inoculation is essential, ensuring that a high and effective rhizobial population is available in the rhizosphere of the plant [36]. The use of Rhizobium inoculants in legumes is the oldest agro-biotechnological application [37]. Several reports demonstrated significant improvement of yield and yield components in faba bean with Rhizobium inoculation [29,38-40].

Generally, the common practice of faba bean cultivation in Egypt is planting the seeds without inoculation. Therefore, most farmers depend on application of high levels of chemical fertilizers to supply $\mathrm{N}$ to plants, particularly under sandy soil conditions with low fertility nature. Since biological $\mathrm{N}_{2}$ fixation is not active at early stages of plant growth, especially under low fertility soils, a starter $\mathrm{N}$-dose $\left(48 \mathrm{Kg} \mathrm{N} \cdot \mathrm{ha}^{-1}\right)$ was applied in this study to enhance plant growth and eventually improve the grain yield production. The application of a starter N-dose with the rate of $48 \mathrm{Kg} \mathrm{N} \cdot \mathrm{ha}^{-1}$ was previously reported to increase nodulation and nitrogen fixation of faba bean under Egyptian soil conditions [41]. In another study, an amount of $40 \mathrm{~kg} \mathrm{~N} \cdot \mathrm{ha}^{-1}$ was used as a starter N-dose by [42], when they measured the field performance of rhizobial inoculants for some important legumes (lentils, soybeans, faba beans, and peanuts) in Egypt under both clay loam Nile Delta soils and virgin sandy soils. Our results are consistent with previous studies which have reported that the application of an amount of $\mathrm{N}$ fertilizer enhances nodulation of different legume crops [33,43,44].

In the present study, we reported the potential use of Rhizobium/Agrobacterium inoculants as a powerful alternate source of $\mathrm{N}$ in low nutrient ecosystems. Under greenhouse conditions (Table 2), all strains nodulated faba bean cultivar Giza 843. Out of the tested strains, eight strains (NGB-FR 39, $62,65,70,107,126,128$, and 140) could establish an effective nitrogen fixation association with this cultivar, producing a dry weight and shoot $\mathrm{N}$ content significantly higher than those obtained by the uninoculated control ( $\mathrm{T}$ ) with $48 \mathrm{Kg} \mathrm{N} \cdot \mathrm{ha}^{-1}$. Previous studies have identified that there are often strong relationships between shoot dry matter and the amount of $\mathrm{N}_{2}$ fixed $[45,46]$.

Under field conditions, growth and grain yield of faba bean increased significantly in response to inoculation with the most effective rhizobial strains (Tables 3-6). Increases in $\mathrm{N}_{2}$ fixation translated to greater grain $\mathrm{N}$ concentration, and therefore resulted in increased $\mathrm{N}$ export from the field at harvest. In the first season (2012/2013), faba bean inoculated with strains A. tumefaciens NGB-FR 62 and Rlv NGB-FR 126 showed significant increases in seed yield $(44 \%-47 \%)$ and seed N-yield $(58 \%-61 \%)$, respectively, relative to the uninoculated control $(\mathrm{T})$. While, in the second growing season (2013/2014), inoculation with strains Rlv NGB-FR 70 and Rlv NGB-FR 126 produced significant increases in seed yield $(69 \%-81 \%)$ and seed N-yield (85\%-94\%), respectively, over the uninoculated control (T). These results are in line with previous report that was published by [29]. They found that in Australia, at sites without soil rhizobia, faba bean grain yield and total grain N increased by $59 \%$ and $132 \%$, respectively, due to different inoculation rates.

Unexpectedly, in the first season (2012/2013), faba bean plants inoculated by A. tumefaciens strain NGB-FR 39 and Rlv strain NGB-FR 70 showed significantly less $\mathrm{N}$ uptake compared to the uninoculated control (T) with $48 \mathrm{~kg} \mathrm{~N} \cdot \mathrm{ha}^{-1}$ (Table 3). This trend was also observed in regards to the final seed $\mathrm{N}$-yield parameter (Table 4). This could be due to the presence of effective indigenous rhizobia or highly competitive but ineffective indigenous strains [47]. Our results are consistent with those published by [48], who reported that $\mathrm{N}$ uptake and $\mathrm{N}_{2}$ fixation response to indigenous soil rhizobia in regards to uninoculated cowpea plants surpassed those of inoculated treatments.

A. tumefaciens were previously isolated from the root nodules of several tropical legumes [49]; Phaseolus vulgaris [50], Sesbania spp. [51], and Vicia faba [20,52]. The ability of A. tumefaciens to nodulate 
legumes roots may be attributed to the possession of a transferred Sym plasmid which enabled them to form root nodules and fix nitrogen symbiotically [53]. However, many Agrobacterium strains isolated from root nodules failed to re-nodulate their original hosts [50,54], which makes Agrobacterium a poor choice for legume inoculation [55]. On the contrary, our results revealed the highly symbiotic stability of tested local $A$. tumefaciens strains to nodulate faba bean roots under both greenhouse and field experiments. The stability of nodulating machinery of Agrobacterium strains with soybean was recently reported [33].

Data presented in this study showed that the increase in seed yield in response to rhizobial inoculation was variable depending upon the strain type and climatic conditions of the cropping year. Similar findings were previously reported on soybean by $[33,56]$. The increments in seed yields in the full $\mathrm{N}$-fertilized plots (TN) and/or inoculated plots, in relation to the uninoculated non-N fertilized plots $\left(\mathrm{T}_{\mathbf{0}}\right)$ controls indicate that, in these soils, nitrogen is a limiting factor, and that crop yields could be strongly improved by means of inoculation or fertilization. However, we found that response to inoculation with the best rhizobial strains was greater than the full $\mathrm{N}$ fertilization $\left(96 \mathrm{Kg} \mathrm{N} \cdot \mathrm{ha}^{-1}\right)$. This study demonstrated the highest potential of rhizobial inoculation as successful alternates of chemical N fertilizers, where effective inoculation with Rlv NGB-FR 126 showed significant increments in the final grain yield ( $35 \%-48 \%)$ and grain $\mathrm{N}$-yield $(34 \%-49 \%)$ compared to the inorganic $\mathrm{N}$-fertilized treatments (TN) over the two cropping seasons, respectively. Our results showed that faba bean inoculation could effectively reduce the need of applied inorganic N-fertilizers while achieving higher grain yield. These findings are in line with those published by [36], who reported that, in a field experiment, inoculation of lentil by Rhizobium strains Lt 29 increased seed yield by $59 \%$ while $\mathrm{N}$ fertilizer ( $50 \mathrm{~kg}$ urea ha ${ }^{-1}$ ) enhanced yields by $40 \%$ over the uninoculated non-fertilized control. Our results are also in agreement with another study [56] which indicated that inoculated soybean under field conditions produced higher or not significantly different seed yields and seed $\mathrm{N}$-yield than the fertilized uninoculated control with $200 \mathrm{~kg} \mathrm{~N} \cdot \mathrm{ha}^{-1}$.

\section{Conclusions}

Field experiments conducted through the two successive growing seasons have demonstrated that nodulation, total $\mathrm{N}$ uptake, and faba bean yield and yield components could be significantly improved through the combined use of Rhizobium/Agrobacterium inoculations and starter $\mathrm{N}$ application (48 kg N.ha ${ }^{-1}$ ) under low fertility sandy soil conditions. Effective inoculation with strain Rlv NGB-FR 126 reduced $50 \%$ of the applied chemical N-fertilizers, while maintaining faba bean productivity at levels significantly higher than those that resulted from having added inorganic $\mathrm{N}$ inputs $\left(96 \mathrm{~kg} \mathrm{~N} \cdot \mathrm{ha}^{-1}\right)$. The results of this study indicate the possibility of using this strain for the development of commercial faba bean inoculants and for achieving better crop yields with reduced usage of $\mathrm{N}$ fertilization.

Acknowledgments: This work has been financed by Science and Technology Development Fund (STDF), Egypt, project ID: STDF 901.

Author Contributions: S.H.Y. designed, conducted the field experiments, analyzed the data and wrote the manuscript. F.H.A. and S.H.Y. prepared the bacterial formulations. S.A.S. conceived the research and revised the manuscript.

Conflicts of Interest: The authors declare no conflict of interest.

\section{References}

1. Sillero, J.C.; Villegas-Fernández, A.M.; Thomas, J.; Rojas-Molina, M.M.; Emeran, A.A.; Fernández-Aparicio, M.; Rubiales, D. Faba bean breeding for disease resistance. Field Crops Res. 2010, 115, 297-307. [CrossRef]

2. Nadal, S.; Suso, M.; Moreno, M. Management of Vicia faba genetic resources: Changes associated to the selfing process in the major, equina and minor groups. Genet. Resour. Crop Evol. 2003, 50, 183-192. [CrossRef] 
3. Nassib, A.M.; Khalil, S.A.; Hussein, A.H.A. Faba bean production and consumption in Egypt. In Present Status and Future Prospects of Faba Bean Production and Improvement in the Mediterranean Countries; Cunero, J.I., Saxena, M.C., Eds.; CIHEAM: Zaragoza, Ethiopia, 1991; pp. 127-131.

4. FAOSTAT. Food and Agriculture Organizations of the United Nations: Statistics Division. 2015. Available online: http:/ / faostat3.fao.org/browse/Q/QC/E (accessed on 21 May 2016).

5. Abdel-Monaim, M.F. Improvement of biocontrol of damping-off and root rot/wilt of faba bean by salicylic acid and hydrogen peroxide. Mycobiology 2013, 41, 47-55. [CrossRef] [PubMed]

6. GRDC, Grains Research and Development Corporation. 2014. Available online: https://grdc.com.au/ Research-and-Development/GRDC-UpdatePapers/2014/07/Faba-bean-marketing-and-the-Middle-East (accessed on 2 June 2016).

7. Hegab, A.S.A.; Fayed, M.T.B.; Hamada, M.M.A.; Abdrabbo, M.A.A. Productivity and irrigation requirements of faba-bean in North Delta of Egyptin relation to planting dates. Ann. Agric. Sci. 2014, 59, 185-193.

8. Zahran, H.H. Rhizobium-legume symbiosis and nitrogen fixation under severe conditions and in an arid climate. Microbiol. Mol. Biol. Rev. 1999, 63, 968-989. [PubMed]

9. Malagnoux, M. Arid land forest of the world: Global environmental perspectives. Forestry Department (FAO), 2007. Available online: http://www.fao.org/3/a-ah836e.pdf (accessed on 28 April 2016).

10. Mmbaga, G.W.; Mtei, K.M.; Ndakidemi, P.A. Extrapolations on the use of Rhizobium inoculants supplemented with phosphorus (P) and potassium (K) on growth and nutrition of legumes. Agric. Sci. 2014, 5, 1207-1226. [CrossRef]

11. Ladha, J.K.; Padre, A.T.; Punzalan, G.C.; Castillo, E.; Singh, U.; Reddy, C.K. Nondestructive estimation of shoot nitrogen in different rice genotypes. Agron. J. 1998, 90, 33-40. [CrossRef]

12. Lowe, P.; Baldock, D. Integration of environmental objectives into agricultural policy making. In $C A P$ Regimes and the European Countryside: Prospects for Integration between Agricultural, Regional and Environmental Policies; Brouwer, F., Lowe, P., Eds.; CABI Publishing: Wallingford, UK, 2000; pp. 31-54.

13. Peoples, M.B.; Herridge, D.F.; Ladha, J.K. Biological nitrogen fixation: An efficient source of nitrogen for sustainable agricultural production? Plant Soil 1995, 174, 3-28. [CrossRef]

14. Herridge, D.F.; Peoples, M.B.; Boddey, R.M. Global inputs of biological nitrogen fixation in agricultural systems. Plant Soil 2008, 311, 1-18. [CrossRef]

15. Van Berkum, P.; Beyene, D.; Vera, F.T.; Keyser, H.H. Variability among Rhizobium strains originating from nodules of Vicia faba. Appl. Environ. Microbiol. 1995, 61, 2649-2653. [PubMed]

16. Allen, O.N.; Allen, E.K. The Leguminosae-A Source Book of Characteristics, Uses and Nodulation; Macmillan Publishers Ltd.: London, UK, 1981.

17. Tian, C.F.; Wang, E.T.; Wu, L.J.; Han, T.X.; Chen, W.F.; Gu, C.T.; Gu, J.G.; Chen, W.X. Rhizobium fabae sp. nov., a bacterium that nodulates Vicia faba. Int. J. Syst. Evol. Microbiol. 2008, 58, 2871-2875. [CrossRef] [PubMed]

18. Saïdi, S.; Ramírez-Bahena, M.H.; Santillana, N.; Zúñiga, D.; Álvarez-Martínez, E.; Peix, A.; Mhamdi, R.; Velázquez, E. Rhizobium laguerreae sp. nov. nodulates Vicia faba on several continents. Int. J. Syst. Evol. Microbiol. 2014, 64, 242-247. [CrossRef] [PubMed]

19. Tian, C.F.; Wang, E.T.; Han, T.X.; Sui, X.H.; Chen, W.X. Genetic diversity of rhizobia associated with Vicia faba in three ecological regions of China. Arch. Microbiol. 2007, 188, 273-282. [CrossRef] [PubMed]

20. Youseif, S.H.; Abd El-Megeed, F.H.; Ageez, A.; Cocking, E.; Saleh, S.A. Phylogenetic multilocus sequence analysis of native rhizobia nodulating faba bean (Vicia faba L.) in Egypt. Syst. Appl. Microbiol. 2014, 37, 560-569. [CrossRef] [PubMed]

21. Lindemann, W.C.; Glover, C.R. Nitrogen Fixation by Legumes. Guide A-129; Cooperative Extension Service, College of Agriculture and Home Economics New Mexico State University: New Mexico, NM, USA, 2003; pp. 1-4. Available online: http://aces.nmsu.edu/pubs/_a/a-129.pdf (accessed on 25 May 2016).

22. Smil, V. Nitrogen in crop production: An account of global flows. Glob. Biogeochem. Cycles 1999, 13, 647-662. [CrossRef]

23. Abdel-Daiem, M.; Hassan, M.F.; Hamdi, Y.A.; Abdel-Ghaffar, A.S. Nitrogen fixation and yield of faba bean, lentil and chickpea in response to selected agricultural practices in Egypt. In World Crops: Cool Season Food Legumes; Summerfield, R.J., Ed.; Kluwer Academic Publishers: London, UK, 1988; pp. 189-204.

24. Abdel-Ghaffar, A.S. Effect of edaphic factors on biological nitrogen fixation in Vicia faba under Egyptian field conditions. In Nitrogen Fixation by Legumes in Mediterranean Agriculture; Beck, D.P., Materon, L.A., Eds.; Martinus Nijhoff Publishers: Dordrecht, The Netherlands, 1988; pp. 325-326. 
25. Denton, M.D.; Coventry, D.R.; Bellotti, W.D.; Howieson, J.G. Distribution, abundance and symbiotic effectiveness of Rhizobium leguminosarum bv. trifolii from alkaline pasture soils in South Australia. Aust. J. Exp. Agric. 2000, 40, 25-35. [CrossRef]

26. Ballard, R.A.; Charman, N.; McInnes, A.; Davidson, J.A. Size, symbiotic effectiveness and genetic diversity of field pea rhizobia (Rhizobium leguminosarum bv. viciae) populations in South Australian soils. Soil. Biol. Biochem. 2004, 36, 1347-1355. [CrossRef]

27. Evans, J. An evaluation of potential Rhizobium inoculant strains used for pulse production in acidic soils of southeast Australia. Aust. J. Exp. Agric. 2005, 45, 257-268. [CrossRef]

28. Hungria, M.; Franchini, J.C.; Campo, R.J.; Crispino, C.C.; Moraes, J.Z.; Sibaldelli, R.N.R.; Mendes, I.C.; Arihara, J. Nitrogen nutrition of soybean in Brazil: Contributions of biological $\mathrm{N}_{2}$ fixation and $\mathrm{N}$ fertilizer to grain yield. Can. J. Plant Sci. 2006, 86, 927-939. [CrossRef]

29. Denton, M.D.; Pearce, D.J.; Peoples, M.B. Nitrogen contributions from faba bean (Vicia faba L.) reliant on soil rhizobia or inoculation. Plant Soil 2013, 365, 363-374. [CrossRef]

30. Vincent, J.M. A Manual for the Practical Study of Root-Nodule Bacteria (International Biological Programme, Handbook No. 15); Blackwell Scientific: Oxford, UK, 1970; p. 164.

31. Page, A.L.; Miller, R.H.; Keeney, D.R. Method of Soil Analysis. Part 2-Chemical and Microbiological Properties; American Society of Agronomy \& Soil Science Society of America: Madison, WI, USA, 1982.

32. Saleh, S.A.; Mekhemar, G.A.A.; Abo El-Soud, A.A.; Ragab, A.A.; Mikhaeel, F.T. Survival of Azorhizobium and Azospirillum in different carrier materials: Inoculation of wheat and Sesbania rostrata. Bull. Fac. Agric. Univ. Cairo 2001, 52, 319-338.

33. Youseif, S.H.; Abd El-Megeed, F.H.; Khalifa, M.A.; Saleh, S.A. Symbiotic effectiveness of Rhizobium (Agrobacterium) compared to Ensifer (Sinorhizobium) and Bradyrhizobium genera for soybean inoculation under field conditions. Res. J. Microbiol. 2014, 9, 151-162. [CrossRef]

34. Snedecor, G.W.; Cochran, W.G. Statistical Methods, 7th ed.; Iowa State University Press: Ames, IA, USA, 1980; pp. 255-269.

35. Freiberg, C.; Fellay, R.; Bairoch, A.; Broughton, W.J.; Rosenthal, A.; Perret, X. Molecular basis of symbiosis between Rhizobium and legumes. Nature 1997, 387, 394-401. [CrossRef] [PubMed]

36. Tena, W.; Wolde-Meskel, E.; Walley, F. Symbiotic efficiency of native and exotic Rhizobium strains nodulating Lentil (Lens culinaris Medik.) in soils of southern Ethiopia. Agronomy 2016, 6. [CrossRef]

37. Lindström, K.; Murwira, M.; Willems, A.; Altier, N. The biodiversity of beneficial microbe host mutualism: The case of rhizobia. Res. Microbiol. 2010, 161, 453-463. [CrossRef] [PubMed]

38. Elsheikh, E.A.E.; Elzidany, A.A. Effects of Rhizobium inoculation, organic and chemical fertilizers on yield and physical properties of faba beanseeds. Plant Foods Hum. Nutr. 1997, 51, 137-144. [CrossRef] [PubMed]

39. Amanuel, G.; Kühne, R.F.; Tanner, D.G.; Vlek, P.L.G. Biological nitrogen fixation in faba bean (Vicia faba L.) in the Ethiopian highlands as affected by P fertilization and inoculation. Biol. Fertil. Soils 2000, 32, 353-359. [CrossRef]

40. Argaw, A. Characterization of symbiotic effectiveness of rhizobia nodulating faba bean (Vicia faba L.) isolated from central Ethiopia. Res. J. Microbiol. 2012, 7, 280-296. [CrossRef]

41. Abdel-Ghaffar, A.S. Nodulation problems and response to inoculation. In First OAU/STRC International African Conference on Bio-Fertilizers, Cairo, Egypt, 22-26 March 1982; 1982.

42. Moawad, H.; El-Din, S.B.; Khalfallah, M.A. Field performance of rhizobial inoculants for some important legumes in Egypt. In Nitrogen Fixation by Legumes in Mediterranean Agriculture; Beck, D.P., Materon, L.A., Eds.; ICARDA: Aleppo, Syria, 1989; pp. 235-244.

43. Daba, S.; Haile, M. Effects of rhizobial inoculant and nitrogen fertilizer on yield and nodulation of common bean. J. Plant Nutr. 2000, 23, 581-591. [CrossRef]

44. Argaw, A.; Tsigie, A. Indigenous rhizobia population influences the effectiveness of Rhizobium inoculation and need of inorganic $\mathrm{N}$ for common bean (Phaseolus vulgaris L.) production in eastern Ethiopia. Chem. Biol. Technol. Agric. 2015, 2. [CrossRef]

45. Peoples, M.B.; Brockwell, J.; Herridge, D.F.; Rochester, I.J.; Alves, B.J.R.; Urquiaga, S.; Boddey, R.M.; Dakora, F.D.; Bhattarai, S.; Maskey, S.L.; et al. The contributions of nitrogen fixing crop legumes to the productivity of agricultural systems. Symbiosis 2009, 48, 1-17. [CrossRef]

46. Unkovich, M.J.; Baldock, J.; Peoples, M.B. Prospects and problems of simple linear models for estimating symbiotic $\mathrm{N}_{2}$ fixation by crop and pasture legumes. Plant Soil 2010, 329, 75-89. [CrossRef] 
47. Samuel, M.; Herrmann, L.; Pypers, P.; Matiru, V.; Mwirichia, R.; Lesueur, D.D. Potential of indigenous bradyrhizobia versus commercial inoculants to improve cowpea (Vigna unguiculata L. walp.) and green gram (Vigna radiata L. wilczek.) yields in Kenya. Soil Sci. Plant Nutr. 2012, 58, 750-763.

48. Aliyu, I.A.; Yusuf, A.A.; Abaidoo, R.C. Response of grain legumes to rhizobial inoculation in two Savanna soils of Nigeria. Afr. J. Microbiol. Res. 2013, 7, 1332-1342.

49. De Lajudie, P.; Willems, A.; Nick, G.; Mohamed, T.S.; Torck, U.; Filai-Maltouf, A.; Kersters, K.; Dreyfus, B.; Lindstrom, K.; Gillis, M. Agrobacterium bv. 1 strains isolated from nodules of tropical legumes. Syst. Appl. Microbiol. 1999, 22, 119-132. [CrossRef]

50. Mhamdi, R.; Mrabet, M.; Laguerre, G.; Tiwari, R.; Aouani, M.E. Colonization of Phaseolus vulgaris nodules by Agrobacterium-like strains. Can. J. Microbiol. 2005, 51, 105-111. [CrossRef] [PubMed]

51. Cummings, S.P.; Gyaneshwar, P.; Vinuesa, P.; Farruggia, F.T.; Andrews, M.; Humphry, D.; Elliott, G.N.; Nelson, A.; Orr, C.; Pettitt, D.; et al. Nodulation of Sesbania species by Rhizobium (Agrobacterium) strain IRBG74 and other rhizobia. Environ. Microbiol. 2009, 11, 2510-2525. [CrossRef] [PubMed]

52. Tiwary, B.N.; Prasad, B.; Ghosh, A.; Kumar, S.; Jain, R.K. Characterization of two novel biovar of Agrobacterium tumefaciens isolated from root nodules of Vicia faba. Curr. Microbiol. 2007, 55, 328-333. [CrossRef] [PubMed]

53. Sawada, H.; Kuykendall, L.D.; Young, J.M. Changing concepts in the systematics of bacterial nitrogen-fixing legume symbionts. J. Gen. Appl. Microbiol. 2003, 49, 155-179. [CrossRef] [PubMed]

54. Wang, L.L.; Wang, E.T.; Liu, J.; Li, Y.; Chen, W.X. Endophytic occupation of root nodules and roots of Melilotus dentatus by Agrobacterium tumefaciens. Microb. Ecol. 2006, 52, 436-443. [CrossRef] [PubMed]

55. Shamseldin, A.A.; Vinuesa, Y.P.; Thierfelder, H.; Werner, D. Rhizobium etli and Rhizobium gallicum nodulate Phaseolus vulgaris in Egyptian soils and display cultivar-dependent symbiotic efficiency. Symbiosis 2005, 38, 145-161.

56. Albareda, M.; Rodríguez-Navarro, D.N.; Temprano, F.J. Use of Sinorhizobium (Ensifer) fredii for soybean inoculants in South Spain. Europ. J. Agron. 2009, 30, 205-211. [CrossRef]

(C) 2017 by the authors; licensee MDPI, Basel, Switzerland. This article is an open access article distributed under the terms and conditions of the Creative Commons Attribution (CC-BY) license (http://creativecommons.org/licenses/by/4.0/). 